\title{
Two ways to form a portmanteau: Evidence from ellipsis
}

\author{
Neil Banerjee*
}

\begin{abstract}
Bengali negation forms a portmanteau in two cases: with present tense existential copulas and with all perfects. However, ellipsis of the complement of negation treats these two portmanteaux differently. While the negative perfect can be separated by ellipsis into sentential negation and a silenced perfect, the negative present existential cannot be likewise split, even though ellipsis of copulas is generally permitted. This project proposes the existence of two different ways to form portmanteaux and shows that ellipsis deletion is derivationally timed differently with respect to each in order to capture the patterns of elliptical divisibility.
\end{abstract}

Keywords. portmanteau; ellipsis; morphology

1. Introduction. A portmanteau is a non-decomposable string of segments which nonetheless seems to correspond to distinct pieces of meaning. If two pieces of meaning are sometimes realised by separate lexical items, but at other times by a single non-decomposable one, we may wonder how the single portmanteau comes to correspond to pieces of meaning that are in principle expressible separately. Several theories have been advanced in the literature for how portmanteau come about, including through dedicated morphological operations like Fusion (Noyer 1992), two cases of contextual allomorphy (Trommer 1999), and the operation that associates phonology with abstract features itself (Caha 2009; Radkevich 2010). This paper uses ellipsis to show at least two distinct operations to form portmanteaux must exist in the grammar.

The emprical focus of the paper is on Bengali, an eastern Indo-Aryan language, which has several portmanteau involving negation and some verbal component. Since ellipsis of the complement of negation is independently permitted in Bengali, we can create a situation where the ingredients of a portmanteau are structurally present, but separate by an ellipsis boundary. Negative copula portmanteaux in this situation block ellipsis, but negative perfect portmanteaux in the same situation are split by ellipsis. Since the same ellipsis operation is involved in both cases, the different elliptical divisibility behaviours of the portmanteaux must be a product of the portmanteau-forming mechanism responsible for each.

A timing analysis is presented to account for the elliptical divisibility facts. In brief, the indivisible portmanteau form before ellipsis occurs, and so can block it. Divisible portmanteaux only form after ellipsis and so are bled by it. The analysis is implemented in the Distributed Morphology framework Halle \& Marantz (1993, 1994). Fusion and contextual allomorphy used as the example mechanisms for the two different types of portmanteaux, though nothing hinges on these being the specific mechanisms as long as the relative timing is obeyed by the proposed mechanisms.

The rest of the paper is structured as follows. Section 2 presents the data from Bengali negation, copulas, and perfects. Section 3 analyses the ellipsis construction to argue that even in the divisible portmanteau case, the ingredients to form the portmanteau are present inside

\footnotetext{
* Thanks to Adam Albright, Bronwyn Bjorkman, Sabine Iatridou, and Norvin Richards for discussion, and Debal Banerjee, Sagarika Banerjee, Shoma Bhattacharya, Amy Csete, Pieter Dhondt, Ankana Saha, and Dóra Kata Takács for judgements. This research was supported by the Social Sciences and Humanities Research Council of Canada Doctoral Fellowship 752-2017-0069. The standard disclaimers apply. Author: Neil Banerjee, Massachusetts Institute of Technology (neilb@mit.edu).
} 
the ellipsis site, and so the portmanteau is expected to form, just like in the indivisible case. Section 4 presents the timing analysis that accounts for the observed elliptical divisibility patterns, and shows an implementation of the analysis. Section 5 discusses alternative implementations, extensions to other languages, predictions of the approach, and speculates on how the elliptical divisibility of a given portmanteau may be learned without reference to elliptical examples. Section 6 discusses next steps and concludes.

2. Data. Sentential negation in Bengali usually involves adding na to the end of the inflected verb as in (1). However, negative perfects and negative copulas cannot be negated in this way, as shown in (2-a) and (3-a). Instead, they both involve specific negative portmanteaux as in (2-b) and (3-b).
a. mach kha-i
fish eat-1
'I eat fish.'
b. mach kha-i na
fish eat-1 NEG
'I do not eat fish.'

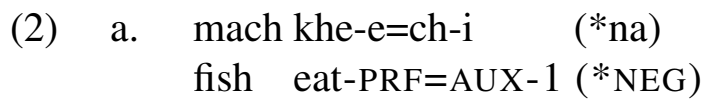

'I have (*not) eaten.'

b. mach kha-i ni

fish eat-1 NEG.PRF

'I haven't eaten fish.'

(3) a. menu-te mach ach-e (*na)

menu-LOC fish be-3 (*NEG)

'There is (*not) fish on the menu.'

b. menu-te mach nei

menu-LOC fish NEG.be

'There isn't fish on the menu.'

The negative perfect portmanteau replaces the perfect marker $-e$ and sentential negation. The auxiliary present in the affirmative perfect is also absent in negative perfects. The negative copula portmanteau replaces the present tense copula, negation, and agreement. Past tense copulas are negated the way most other verbs are, with a separate negative marker as in (4).
menu-te mach chi-l-o
(na)
MENU-LOC FISH BE-PST-3.PST (NEG)
'There was (not) fish on the menu.'

These negative portmanteau can be made to interact with ellipsis because Bengali independently permits ellipsis of the complement of negation. When this ellipsis tries to split the two negative portmanteaux, we see two different patterns. Ellipsis can split a negative perfect portmanteau into sentential negation and a silenced perfect as in (5-a), but it cannot likewise split a negative copula into sentential negation and a silenced copula as in (5-b). 

a. lal alo-ța jol-e=ch-e, kintu nil-ța na
red light-CL light.ITR-PRF=AUX-3, but blue-CL NEG
'The red light has turned on, but not the blue one.'
b. *menu-te amis pd to ach-e, kintu mach na menu-LOC non-vegetarian items PRT be-3, but fish NEG
'There are non-vegetarian item on the menu, but not fish.'

The inability to elide the copula is not a general property of copulas, since the non-portmanteau forming past copula can be elided under negation as in (6). It is only the portmanteau copula which appears to block ellipsis.

menu-te amis pd to chi-l-o, kintu mach na
menu-LOC non-vegetarian items PRT be-PST-3.PST, but fish NEG
'There were non-vegetarian item on the menu, but not fish.'

In summary, Bengali negation forms a portmanteau in two cases: negative perfects and negative present copulas. While negative perfects are elliptically divisible, negative copula are not.

3. Analysis. In this section, properties of the ellipsis will be investigated to determine whether the differences in elliptical divisibility can be analysed as a result of properties of the ellipsis operation. An instance of ellipsis can be characterized by the position of the ellipsis site and the structure inside it. We will see that on both counts, ellipsis of a copula and of a perfect pattern alike.

Suppose that the position of the ellipsis site were different in (5-b) and (5-a). In particular, if (5-a) involved constituent negation on the object rather than sentential negation, we would not expect a portmanteau negative perfect to form and possibly block ellipsis. This hypothesis can be tested with Negative Polarity Items (NPIs), since they must be in the scope of negation to be licensed. Consider example (7).

$$
\begin{aligned}
& \text { ònek bar ilis khe-e=ch=i, kintu kòkkhono magur na } \\
& \text { many times hilsa eat-PRF=AUX-1, but ever catfish NEG }
\end{aligned}
$$

'I have eaten hilsa many times, but never catfish.'

The VP-level NPI kòkkhono, 'ever', requires negation to scope over it to be licensed. If the negation visible in (7) were constituent negation on the remnant, it would be too low, since the remnant is the object of the elided verb. From the fact that the NPI is licensed in (7), we can conclude that the visible negation is sentential negation, not constituent negation on the remnant. For posterity, note that the same NPI is licensed with ellipsis of a copula as well, as shown in (8).

$$
\begin{aligned}
& \text { ònek din bihar-e chi-l-am, kintu kòkkhono pața-te na } \\
& \text { many day Bihar-LOC be-PST-1.PST, but ever Patna-LOC NEG } \\
& \text { 'I was in Bihar for a long time, but never in Patna.' }
\end{aligned}
$$

Although the position of the ellipsis sites is the same in both cases, it is still possible that the amount of structure they contain differs. In particular, if (5-a) does not contain the structure of a perfect, the ingredients for a portmanteau would not be present, and so no portmanteau would form to block ellipsis. This hypothesis can be tested using the connectivity effects from Merchant (2018), which show evidence for structure inside ellipsis sites. Case matching, where 
the remnant of ellipsis must match in case with its correlate, is shown in (9).

$$
\begin{array}{llll}
\text { masi- }(* \mathrm{r}) \quad \text { khide } & \text { pe-e=ch-e, } & \text { kintu meso- }(* \mathrm{r}) & \text { na } \\
\text { auntie- }(* \text { GEN }) \text { hunger get-PRF=AUX-3, but uncle- }(* \text { GEN }) & \text { NEG }
\end{array}
$$

'Auntie is hungry, but not Uncle.'

The predicate khide paoa 'be hungry' takes a genitive experiencer in Bengali. As (9) shows, even if the predicate is elided, the remnant experiencer must still bear genitive case. This is expected if the ellipsis site contains a silent case assigner, but mysterious otherwise. See Banerjee (under review) for evidence from island sensitivity which further supports the conclusion that the ellipsis site in the complement of negation in Bengali contains articulated structure.

While this shows that the ellipsis sites contain structure, it does not show that the structure is that of a perfect. Since simple pasts also express anteriority like perfects, it is plausible to hypothesize that the parallelism requirements on ellipsis are sufficiently loose that a simple past could be elided under identity with a perfect. If this happened, no portmanteau would be expected to form either, accounting for (5-a). This hypothesis can be tested using the semantic traces of a perfect. As per Iatridou et al. (2001), many languages have perfects with a resulting state requirement, wherein the state described by the perfect must hold until the reference time set by tense. In the case of a present perfect, this means the state that results from the event described must hold until utterance time. The unavailability of the continuation in (10-a), below, shows that the state of the stove being unlit must hold if the state is described using a perfect. Example (10-b) shows that no such requirement exists for the simple past.
a. unun-ța jòl-e ni (\# tai deslai die jala-l-am)
stove-CL light.ITR-3 NEG.PRF (\# so match with light-PST-1.PST)
'The stove hasn't turned on (\# so I lit it with a match).'
b. unun-ta jol-1-o na (tai deslai die jala-l-am)
stove-CL light.ITR-PST-3.PST NEG (so match with light-PST-1.PST)
'The stove didn't turn on (so I lit it with a match).'

If the ellipsis site in an apparent division of a portmanteau negative perfect does not actually contain a perfect, the resulting state requirement should by obviated by ellipsis. Example (11) shows that this is not the case.

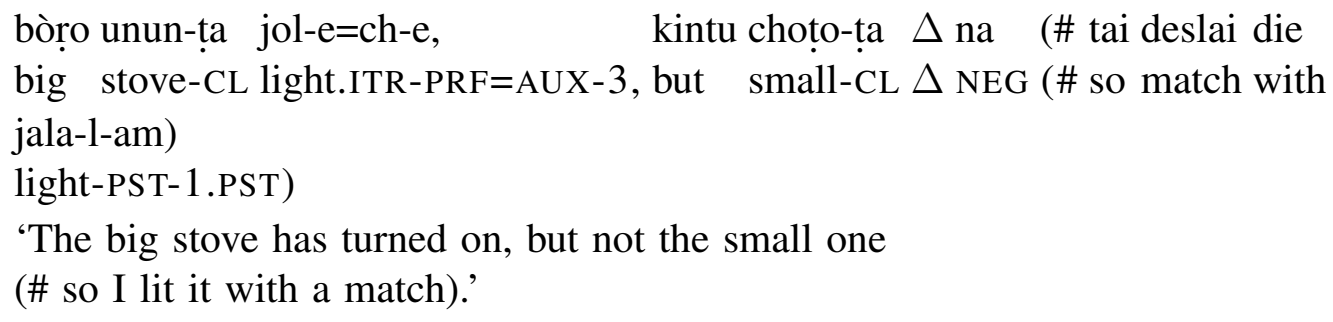

The existence of a resulting state requirement even with ellipsis of a perfect shows that not only does the ellipsis site in example like (5-a) contain structure, but moreover, the structure is that of a perfect. This means that properties of ellipsis are not responsible for the differences in elliptical divisibility between negative perfecst and negative copulas. In both cases, the ellipsis site is at the same structural position, and in the case of the successful splitting of a negative perfect, the ingredients for the portmanteau are present inside and outside the ellipsis site. The source of the variation in elliptical divisibility must be the mechanisms by which 
portmanteaux are formed. If only one such mechanism existed, its interaction with the single ellipsis process could not create two different patterns of divisibility, so we can conclude that there must be two distinct ways to form portmanteaux.

4. Proposal. The differences in elliptical divisibility can be accounted for using a timing analysis. I propose that indivisibile portmanteaux like the negative copula form before ellipsis applies. This way, they are already units when ellipsis happens, and so cannot be split into pieces. Divisible portmanteaux like the negative perfect form after ellipsis applies. Since they are not units at the time of ellipsis, parts of them can be deleted separately. I implement this proposal in the Distributed Morphology Halle \& Marantz (1993, 1994) framework of a Late Insertion model of the morphology. In this model, syntax operates over abstract feature bundles, and a post-syntactic operation called Insertion is responsible for association phonological content (called a vocabulary item) with the abstract features at a given terminal node.

I propose that Fusion (Noyer 1992) underlies indivisible portmanteaux. Fusion takes adjacent terminals as input and replaces them with a single terminal that contains the union of the features of the original terminals. This creates portmanteaux because when Insertion applies later, all of the relevant features are in a single terminal, and so will be associated with a single vocabulary item. I propose that divisible portmanteau are the result of two cases of contextual allomorphy, following Trommer (1999). One of the allomorphs happens to be null, creating the illusion of a single vocabulary item associated with multiple sets of features. In reality, each part of the apparent portmanteau is realised separately. Contextual allomorphy happens at the point of Insertion, so it is ordered after Fusion anyway. All that we need now is a mechanism by which to accomplish ellipsis in between the two.

Murphy (2016) proposes that ellipsis can be accomplished by the operation of Impoverishment, which deletes features on terminals. Normally, this operation is used to delete a subset of the features on a terminal when they are in conflict with or they duplicate features elsewhere. Murphy (2016) proposes that Impoverishment can be total, in the following circumstances:

(12) For any $F, F$ a feature on $L, F \rightarrow \varnothing$ iff there is an $L^{\prime}$ such that $F$ is on $L^{\prime}$ and $L_{F} \subseteq$ $L_{F}^{\prime}$

This states that all of the features on a terminal $L$ can be deleted if they are a subset of the features on a terminal $L^{\prime}$ that can serve as an antecedent to $L .{ }^{1}$

Given these three ingredients, the patterns of elliptical divisibility can be captured if the post-syntactic operations are ordered as follows:

$$
\text { Fusion } \prec \text { Impoverishment } \prec \text { Insertion }
$$

Let us consider each type of portmanteau in turn to see why.

4.1. INDIVISIBLE PORTMANTEAU: FUSION $\prec$ IMPOVERISHMENT. On this proposal, indivisible portmanteau are the result of Fusion, which precedes ellipsis by total Impoverishment. I propose that the following Fusion rule exists in the grammar. ${ }^{2}$

\footnotetext{
${ }^{1}$ Murphy (2016) uses proper subsets as part of the identity requirement, which I relax to subsets instead of proper subsets. He notes in footnote 4 (Murphy 2016; 9) that a weaker identity requirement may be correct, but his cases do not distinguish them. In this case, proper subsets would be too strong, and so I opt for just plain subsets instead.

${ }^{2}$ Fusion is normally defined as operating on sister terminals, so a single rule could not fuse 3 heads. Two rules would be needed instead, but they are collapsed into one for brevity, since the details of what each step of Fusion does on its
} 


$$
[\sqrt{ } \mathrm{BE}][\mathrm{PRS}][\mathrm{NEG}] \rightarrow[\sqrt{ } \mathrm{BE}, \mathrm{PRS}, \mathrm{NEG}]
$$

Given this Fusion rule, consider what happens to portmanteau-forming negative present copula and a non-portmanteau negative past copula. The table below represents the relevant pieces of the antecedent, the ellipsis target, and the result of Fusion for both cases.

$\begin{array}{lll} & \text { Portmanteau } & \text { Non-portmanteau } \\ \text { Antecedent } & {[\sqrt{ } \mathrm{BE}][\mathrm{PRS}]} & {[\sqrt{ } \mathrm{BE}][\mathrm{PST}]} \\ \text { Ellipsis Target } & {[\sqrt{ } \mathrm{BE}][\mathrm{PRS}][\mathrm{NEG}]} & {[\sqrt{ } \mathrm{BE}][\mathrm{PST}][\mathrm{NEG}]} \\ \text { Fusion } & {[\sqrt{ } \mathrm{BE}, \mathrm{PRS}, \mathrm{NEG}]} & - \\ \text { Impoverishment } & - & {[\sqrt{ } \mathrm{BE}][\mathrm{PS}][\mathrm{NEG}]}\end{array}$

Table 1. Fusion and Impoverishment

In the non-portmanteau case, no Fusion rule is triggered. When Impoverishment applies, it finds that there are two terminals with antecedents that contain a superset of their features, namely $[\sqrt{ } \mathrm{BE}]$ and $[\mathrm{PST}]$. It deletes these features, as represented by the strikethroughs. As a result of this, only negation remains in the non-portmanteau case when Insertion subsequently applies. This is a normal application of ellipsis. In the portmanteau case however, Fusion is triggered in the Ellipsis target, and replaces the separate terminals $[\sqrt{ } \mathrm{BE}]$, [PRS], and [NEG] with a single terminal containing the union of the features: $[\sqrt{ } \mathrm{BE}, \mathrm{PRS}, \mathrm{NEG}]$. Notice that when Impoverishment goes to apply, there is no longer any terminal in the antecedent that contains a superset of the features of the ellipsis target. Fusion has applied, and created a node that contains a set of features that are distributed across multiple heads in the antecedent. As a result, Impoverishment cannot apply, and so ellipsis is blocked by the existence of the portmanteau. This captures how elliptically indivisible portmanteaux can block ellipsis by forming prior to ellipsis silencing.

4.2. DiVisible PORTMANTEAU: IMPOVERISHMENT $\prec$ INSERTION. On this proposal, divisible portmanteaux are the result of two cases of contextual allomorphy, which follow Impoverishment. The analysis is that of Banerjee (2020), where the negative perfect $n i$ is actually the exponent of negation in the context of a silent perfect, while the perfect has a zero allomorph in the context of negation. The vocabulary items required are listed in (15).

$$
\begin{array}{ll}
\text { a. } & {[\mathrm{PRF}] \leftrightarrow \varnothing / /_{-}[\mathrm{NEG}]} \\
\text { b. } & {[\mathrm{PRF}] \leftrightarrow-\mathrm{e}} \\
\text { c. } & {[\mathrm{NEG}] \leftrightarrow \mathrm{ni} / /_{-}[\mathrm{PRF}]} \\
\text { d. } & {[\mathrm{NEG}] \leftrightarrow \mathrm{na}}
\end{array}
$$

Given these vocabulary items, consider what happens to a negative perfect undergoing ellipsis. The table below represents the relevant pieces of the antecedent, the ellipsis target, and the result of Impoverishment.

Impoverishment applies to the ellipsis target, and deletes the [PRF] and [PRS] features because they are contained on terminals in the antecedent. Subsequently, when Insertion applies, it can only target [NEG] since the terminals below that have been deleted. The negative perfect

own is not important. 


\begin{tabular}{|c|c|}
\hline Antecedent & $\ldots$ [PRF][PRS] \\
\hline Ellipsis Target & $\ldots$ [PRF $][\mathrm{PRS}][\mathrm{NEG}]$ \\
\hline Impoverishment & $\ldots$ [PRF $][\mathrm{PRS}][\mathrm{NEG}]$ \\
\hline Insertion & $\begin{array}{ll}\ldots & \text { na }\end{array}$ \\
\hline
\end{tabular}

Table 2. Impoverishment and Insertion

allomorph $n i$ cannot be inserted in this case, because the context of Insertion for the vocabulary item in (15-c) is not met. So only the elsewhere vocabulary item in (15-d) can be inserted. The result is an apparently 'split' portmanteau: the expected portmanteau form $n i$ is replaced by the elsewhere negation $n a$ and a silenced perfect. This captures how elliptically divisible portmanteaux are bled by ellipsis.

5. Discussion. This section outlines four areas of discussion that arise from the proposal. Firstly, it demonstrates that the core of the proposal is just the relative timing of the mechanisms of portmanteau formation, and that the exact implementations are less important than their relative timing. Secondly, it discusses examples of the phenomenon in other languages. Thirdly, it evaluates a prediction of the approach, namely, that contextual allomorphy should be bled by ellipsis. Finally, it addresses the question of whether the elliptical divisibility of a portmanteau is determinable from some independent properties or if evidence from an elliptical example is required to determine the divisibility of a portmanteau.

5.1. Alternatives. The key part of the proposal is the relative timing of the formation of indivisible portmanteaux, ellipsis silencing, and divisible portmanteaux. As long as the following order of events holds, the exact mechanism is not crucial.

Indivisible portmanteaux $\prec$ Ellipsis $\prec$ Divisible portmanteaux

For example, Caha (2009); Radkevich (2010); Haugen \& Siddiqi (2016), among others, have noted that Fusion may not be a desirable operation in the grammar. This is because it has a certain lookahead quality to it. Chung (2007) notes that Fusion has to selectively apply to terminals if a portmanteaux exponent exists for the union of the features. But the association with an exponent only happens by the Insertion algorithm, which has to follow Fusion in order to be fed by it. This means that Fusion needs to have access to the possible outputs of a subsequent operation to know whether to apply or not. Instead, these authors, among others, have proposed that Insertion and Fusion can be combined into a single operation that associates exponents with structures larger than single terminals. These Non-Terminal Insertion (NTI) approaches differ on what exactly the target of Insertion is. For Caha (2009) and Radkevich (2010) it is subtrees, while for Svenonius (2016) it is structural spans of adjacent heads, and for Haugen \& Siddiqi (2016) and Ostrove (2018), it is linear spans of adjacent heads. I will briefly sketch an alternative implementation which uses structural spans as in Svenonius (2016), and show that the different elliptical divisibility behaviours can be captured straightforwardly as long as the relative timing analysis is obeyed.

If Fusion and Insertion are combined into a single NTI operation, both divisible and indivisible portmanteau will be the result of the new Insertion operation, although by different mechanisms. Crucially though, this means that ellipsis can no longer be modelled as the result of Impoverishment, because that will precede Insertion and so precede the formation of 
indivisible portmanteaux. Fortunately, alternative approaches to ellipsis exist that are suitable to such an account. Merchant (2001) proposes that ellipsis is the result of an abstract feature [E] on the sister of the ellipsis site. This feature triggers various syntactic, semantic, and postsyntactic effects, which together bring about the phenomenon of ellipsis. Of interest to us is the post-syntactic effects of ellipsis. Suppose that the Insertion algorithm looks as in (17).

Insertion algorithm

1.Target the lowest terminal that is unassociated with a vocabulary item

2.If the target hosts [E], delete its complement

3.Find the largest structural span anchored by the targeted terminal such that there is a single vocabulary items that expones at least as large a subset of the features in the span as separate overt vocabulary items can (c.f. the Insertion Principle from Haugen \& Siddiqi (2016))

4.Associate the vocabulary item with all the terminals in the span

5.Repeat from start until no further Insertion is possible

On this view, indivisible portmanteaux are the result of a single span, as shown below, while divisible portmanteaux are still the result of two cases of contextual allomorphy.

$$
\langle[\sqrt{ } \mathrm{BE}][\mathrm{PRS}][\mathrm{NEG}]\rangle \leftrightarrow \text { nei }
$$

Suppose such a span exists in a structure, with [NEG] hosting an [E] feature. This is the setup for an attempt to split the portmanteau nei. As per the algorithm, Insertion will first target the root, and finding a span that can be associated with nei will do so. At that point, notice that [NEG] has been associated with an exponent (along with several other heads) without being directly targeted for Insertion. As per the algorithm, Insertion will then target the next lowest terminal unassociated with a vocabulary item. In particular since [NEG] is already associated with nei at this point, Insertion will not target it and so it will never read the [E] feature and trigger deletion. The result of this algorithm is that an $[\mathrm{E}]$ feature that is in the middle of a span corresponding to a single vocabulary item will never be read by Insertion and so never trigger ellipsis. In other words, portmanteaux formed by spanning are elliptically indivisible. Divisible portmanteaux remain the result of two cases of contextual allomorphy, and so the analysis of their divisibility remains much the same. Since association with a vocabulary item follows deletion due to [E], if a [NEG] head is not part of a span portmanteau and hosts [E], its complement will be deleted before it is associated with a vocabulary item.

Notice that though the details of this implementation differ significantly from the first one discussed, the crucial timing relations are still maintained. Ellipsis is triggered when Insertion reads the sister of the ellipsis site, and portmanteaux by span, which are indivisible, form when Insertion reads the lowest terminal in the span. So, if ellipsis tries to 'split' an indivisible portmanteaux, the [E] feature will be somewhere above the lowest node in the span, and Insertion would create the portmanteau before it could bring about ellipsis. For any given structure, possble ellipsis of a span portmanteau will still follow the formation of the indivisible portmanteaux. Divisible portmanteaux only exist when all of their parts have been associated with phonology, which crucially means after their highest feature is associated with a vocabulary item. But ellipsis will be triggered before the vocabulary item is chosen for the highest head 
participating in the portmanteaux, and so ellipsis will precede the formation of divisible portmanteaux.

So, while the details of the implementation are not crucial, as long as indivisible portmanteaux form before ellipsis and divisible portmanteaux form after, the differing patterns in divisibility can be captured. Since the two portmanteaux forming operations are derivationally timed differently with respect to ellipsis, regardless of what their exact mechanism is, they must be distinct operations: there are (at least) two ways to form portmanteaux.

5.2. Extensions to other LANGUAGes. Neither indivisible portmanteaux, nor divisible portmanteaux are unique to Bengali. For example, the Hungarian negative indicative 3rd person present copula nincs(enek) is elliptically indivisible, just like Bengali nei. Table 3 shows the paradigm for present and past tense affirmative and negative copulas in Hungarian. The cells in bold represent the portmanteau negative copulas, which appear in place of the analytically expected *nem van(nak).

\begin{tabular}{l|l|l|l|l} 
& AFF.PRS & AFF.PST & NEG.PRS & NEG.PST \\
\hline 1SG & vagy-ok & vol-t-am & nem vagy-ok & nem vol-t-am \\
\hline 1 PL & vagy-unk & vol-t-unk & nem vagy-unk & nem vol-t-unk \\
\hline 2SG & vagy- $\varnothing$ & vol-t-ál & nem vagy- $\varnothing$ & nem vol-t-ál \\
\hline 2PL & vagy-tok & vol-t-atok & nem vagy-tok & nem vol-t-atok \\
\hline 3SG & van- $\varnothing$ & vol-t- $\varnothing$ & nincs- $\varnothing$ & nem vol-t- $\varnothing$ \\
\hline 3PL & van-nak & vol-t-ak & nincs-enek & nem vol-t-ak
\end{tabular}

Table 3. Hungarian existential/locative copula paradigm

Like Bengali, Hungarian also permits ellipsis of the complement of negation generally, but ellipsis of a 3rd person present copula under negation is not permitted.
a. Pisti otthon volt, de Ildi nem
Pisti at.home be.3SG.PST, but Ildi NEG
'Pisti was at home, but not Ildi.'
b. Pisti otthon van, de n nem
Pisti at.home be.3SG.PRS, but I NEG
Int: 'Pisti is at home, but not Ildi.'
c. *Pisti otthon van, de Ildi nem
Pisti at.home be.3SG.PRS, but Ildi NEG
Int: 'Pisti is at home, but not Ildi.'

Both ellipsis of a 3rd person past copula as in (19-a) and of a 1st person present copula as in (19-b) is permitted, but ellipsis cannot split nincs into an overt negation and a silenced copula as in (19-c).

And example of a divisible portmanteau comes from the Dutch negative indefinite marker geen, which must appear in place of sentential negation niet and an indefinite article een.

a. Voor de test, mag u niet eten before DEF test, may you NEG eat.INF

'You are not allowed to eat before the test.' 
b. *Voor de test, mag u niet een maaltijd eten before DEF test, may you NEG IDF meal eat.INF

Int: 'You are not allowed to eat a meal before the test.'

c. Voor de test, mag u geen maaltijd eten

before DEF test, may you NEG.IDF meal eat.INF

'You are not allowed to eat a meal before the test.'

Dutch permits ellipsis in the complements of certain root modals, which Aelbrecht (2010) analyses. See Aelbrecht (2010) for detailed discussion showing the existence of structure inside the ellipsis sites. When this modal complement ellipsis applies below sentential negation however, an indefinite is licensed in the ellipsis site. In other words, geen is split into an overt niet and a silenced een in (21).

(21) Baren wil een auto kopen, maar hij kan niet

Baren want.SG IDF car buy.INF, but he can NEG

'Baren wants to buy a car, but he's not able to.'

So we see that both divisible and indivisible portmanteaux exist in various languages, and the analyses put forth for their mechanisms is of relevance to understanding structures available in language more generally.

5.3. A PREDiction about CONTEXtual allomorphy. The implementations discussed both involve modelling divisible portmanteaux as the result of two cases of contextual allomorphy. If this is correct, it predicts that contextual allomorphy should always be bled by ellipsis if the trigger for the alternation is inside an ellipsis site. Recent work by various authors indicates that this prediction is correct quite generally. For, example, consider Irish conditional suppletion, which is bled by responsive ellipsis.

The Donegal and Mayo dialects of Irish have a conditional verbal suffix -feadh, which shows contextual allomorphy for the category of the subject. If the subject is pronominal, the suffix is pronounced $/ \mathrm{hit}^{\mathrm{j}} /$, and otherwise it is pronounced $/ \mathrm{hu} /$. Data in this section come from Bennett et al. (2019). See their paper for discussion on this alternation, and in particular evidence that it is not phonologically governed. If a pronominal subject is elided by responsive ellipsis, the pronunciation of the conditional marker that is on the remnant verb is the default, and not $/ \mathrm{hit}^{\mathrm{j}} \mathrm{H}$, as shown in (22-b)

(22) An gcuirfeadh sé fearg ort?

Q put.COND.DEP 3SG.M anger on.2?

'Would it make you angry?' [lit. Would it put anger on you?]

a. Chuirfeadh sé fearg orm

put.COND.IND 3SG.M anger on.1

$/ \mathrm{xir}^{\mathrm{j}} \mathrm{hit}^{\mathrm{j}} /, * \mathrm{xir}^{\mathrm{j} h u /}$

'It would make me angry.' [lit. It would put anger on me.]

b. Chuirfeadh sé fearg orm

put.COND.IND 3SG.M anger on.1

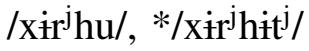

'Yes.' [lit. Would put] 
We see that ellipsis of the trigger, namely the subject, bleeds contextual allomorphy in these Irish dialects. Allomorphy being bled by ellipsis is also argued for in various other languages. See Weisser (2019) for an example from Bavarian, Sailor (forthcoming) for examples from Taiwanese and Verbicarese, and Ronai \& Stigliano (2020) for an example from Hungarian. So analysing divisible portmanteaux as contextual allomorphy is particularly suitable because both behave the same way when interrupted by ellipsis.

5.4. Determining DiVisibiLity. Suppose a linguist or a learner comes across a portmanteau. How do they tell whether it is elliptically divisible or not? Is evidence from an elliptical example required to determine elliptical divisibility? I argue that the locality of the pieces of the portmanteau might be sufficient to determine elliptical divisibility if a stipulation about the default mechanism to store portmanteaux is made.

Consider the divisible negative perfect portmanteau $n i$. It corresponds to the perfect, and to negation, but not to subject agreement, which is shown in (23) to vary.

ami/tumi/o kha-i/o/e ni

I/you/(s)he eat-1/2/3 NEG.PRF

'I/you/(s)he have/has not eaten.'

We can use the Mirror Principle (Baker 1985) to determine the positions of the pieces along the spine. Given that all of the relevant morphemes are suffixes, we can deduce that any affix that is closer to the root on the surface is structurally closer to the root as well. Consider the examples below, which jointly tell us where pieces of the spine are located.

$$
\begin{aligned}
& \text { a. mach khe-e=ch-i } \\
& \text { fish eat-PRF=AUX-1 } \\
& \text { 'I have eaten fish.' } \\
& \text { b. mach kha-i na } \\
& \text { fish eat-1 NEG } \\
& \text { 'I don't eat fish.' }
\end{aligned}
$$

Following Bjorkman (2011), I assume that the auxiliary is not hosted in a projection, but rather inserted late to rescue inflectional morphology that would otherwise be stranded. Given this, (24-a) tells us that the perfect is closer to the root than T+Agr, and (24-b) tells us that negation is further from the root than T+Agr. From these two examples, we can jointly construct the following sketch of the spine for a negative perfect in (23).

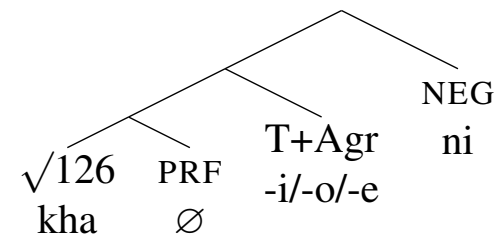

Notice that the two pieces of the portmanteau, PRF and NEG, are separated from each other by the overt T+Agr head. Both Fusion and NTI models of portmanteau have strict locality restrictions. For Fusion, the canonical restriction is that the heads undergoing it must be sisters. In order to Fuse PRF and NEG, PRF would have to move to NEG across T+Agr in violation of the head movement constraint (Travis 1984). NTI requires either a subtree or a span to contain just the pieces of the portmanteau. But in the structure in (25), every span or subtree that 
contains both PRF and NEG also contains the intervening T+Agr. So, neither Fusion nor NTI as canonically defined could have been used to model the indivisible portmanteau, because the locality restrictions required for these mechanisms is not met by the pieces of the portmanteau.

Turning to the indivisible portmanteau, we see that the locality restrictions are met. The indivisible negative present copula does not show any agreement, as in (26).

$$
\begin{aligned}
& \text { ami/tumi/o chobi-te nei } \\
& \text { I/you/(s)he picture-LOC NEG.be } \\
& \text { 'I/you/(s)he am/are/is not in the picture.' }
\end{aligned}
$$

As a result, this could be analysed as the result of Fusion or NTI of all of the heads from the existential/locative copula through to negation, crucially including T+Agr. Underspecification for person for the vocabulary item nei accounts for why no agreement morphology is observed anywhere.

However, the observation that the divisible portmanteau could not have been the result of Fusion/NTI is not enough to be able to determine the divisibility of a portmanteau from the locality of the pieces alone. While a portmanteau with non-local pieces has to be the result of two cases of allomorphy, we have no way to decide how to model a portmanteau where the pieces are local to each other, like indivisible nei. This is where a stipulation becomes necessary if divisibility is to be totally determinable from the locality of the pieces.

Suppose we adopt the stipulation that when faced with a portmanteau, the preferred way to analyse and store it is as a single unit. In other words, the Fusion/NTI approach to portmanteau is the default mechanism for the learner. Only when faced with a portmanteau where the pieces are not sufficiently local will the learner resort to storing the portmanteau as the result of two separate vocabulary items which conspire to create the appearance of a single one. If this stipulation is granted, then divisibility can be totally determined from locality. The stipulation ensures that portmanteau with sufficiently local pieces will be stored as the result of Fusion/NTI, while portmanteau with non-local pieces will be stored as the result of allomorphy.

This stipulation predicts that portmanteau with pieces local to one another will be indivisible, while those with pieces that are not local to one another will be divisible. This appears to be borne out by the data from Hungarian and Dutch. Hungarian nincs corresponds to a copula, present tense, 3rd person agreement, and negation all at once. There are no heads that are expected to intervene between these, and nincs is indeed indivisible. On the other hand Dutch geen corresponds to sentential negation, which is somewhere structurally above the verb, and the indefinite determiner of the object, which is below the verb. The pieces are not structurally local to each other, since at least the verb intervenes, and indeed geen is divisible. The correlation between the locality of the pieces and the divisibility of the portmanteau seems to be well supported, though the source of the correlation is, for now, a stipulation about the preferred mechanism by which portmanteau are stored in the grammar.

6. Conclusion. Bengali has two cases where negation forms a portmanteau with something in the verbal spine below it. One is with perfects, and the other is with present tense existential/locative copulas. Negation also permits ellipsis of its complement, but this ellipsis operation interacts with the two negative portmanteaux in different ways. While the negative perfect portmanteau can be split by ellipsis, leaving only overt negation outside the ellipsis site, the 
negative copula block ellipsis. This is despite the fact that a past tense copula, which does not form a portmanteau with negation, can be elided in the complement of the ellipsis site.

The two different patterns of elliptical divisibility that result from the interaction of ellipsis and portmanteau formation must be the result of either non-uniformity in the ellipsis operation, or the portmanteau operation (or both). In this paper we have seen that the ellipsis sites in both ellipsis of perfects and of copulas is structurally in the same position, and shows evidence for unpronounced structure inside it. This naturally leads to the conclusion that the different divisibility patterns are the result of there being two different ways to form portmanteaux in the grammar. The divisibility patterns can be straightforwardly accounted for by a timing analysis: indivisible portmanteaux form before ellipsis applies and so can block it, while divisible portmanteaux form after ellipsis has applied and so can be bled by it.

This timing analysis was implemented in the Distributed Morphology framework by modelling indivisible portmanteaux through Fusion, divisible portmanteaux through contextual allomorphy, and ellipsis through Impoverishment. In the discussion section, it was shown that this implementation is not the only possibility. As long as the relative timings of ellipsis and the two portmanteau-forming operations are as described in this paper, the exact mechanisms for portmanteau-formation are not crucial to capturing the two different divisibility patterns.

The analysis of divisible portmanteaux as contextual allomorphy was shown to be in accord with recent work showing that contextual allomorphy is bled when the trigger for the alternation is inside an ellipsis site. In addition, both patterns of divisibility were shown to exist in other languages. Bengali provides an interesting test case, only because both types of portmanteau are evident in the same language, with the same ellipsis construction. This makes it easier to demonstrate that there must be distinct portmanteau-forming operations responsible for the alternation, rather that different types of ellipsis.

The discussion concluded with an investigation into how the divisibility of a given portmanteau could be determined without evidence from ellipsis. A correlation between the locality of the pieces of the portmanteau and its elliptical divisibility was observed. Indivisible portmanteau in both Bengali and Hungarian seem to involve pieces that are structurally local to each other, while divisible portmanteau in both Bengali and Dutch seem to involve pieces that are not structurally local. For now, the correlation was accounted for by means of a stipulation preferring to store portmanteau as indivisible if possible. Exploring whether evidence exists for such an approach or whether a different explanation can be given for the source of the correlation between structural locality and elliptical divisibility is left for future work.

\section{References}

Aelbrecht, Lobke. 2010. The syntactic licensing of ellipsis. Amsterdam: John Benjamins. https://doi.org/10.1075/la.149.

Baker, Mark. 1985. The mirror principle and morphosyntactic explanation. Linguistic Inquiry 16(3). 373-415. https://www.jstor.org/stable/4178442.

Banerjee, Neil. 2020. Ellipsis as obliteration: Evidence from Bengali negative allomorphy. Proceedings of the Linguistic Society of America 5(1). 133-143. https://doi.org/10.3765/plsa.v5i1.4692.

Banerjee, Neil. Under review. Bengali negation is allomorphic: Consequences for ellipsis and morphology. 
Bennett, Ryan, Emily Elfner \& James McCloskey. 2019. Prosody, focus, and ellipsis in Irish. Language 95(1). 66-106. https://doi.org/10.1353/lan.2019.0012.

Bjorkman, Bronwyn. 2011. BE-ing default: The morphosyntax of auxiliaries. Cambridge, MA: Massachusetts Institute of Technology dissertation.

Caha, Pavel. 2009. The nanosyntax of case. Tromsø: University of Tromsø dissertation.

Chung, Inkie. 2007. Suppletive negation in Korean and Distributed Morphology. Lingua 117(1). 95-148. https://doi.org/10.1016/j.lingua.2005.10.002.

Halle, Morris \& Alec Marantz. 1993. Distributed Morphology and the pieces of inflection. In Kenneth Hale \& Samuel Jay Keyser (eds.), The view from Building 20, 111-176. Cambridge, MA: MIT Press.

Halle, Morris \& Alec Marantz. 1994. Some key features of Distributed Morphology. In Andrew Carnie \& Heidi Harley (eds.), Papers on phonology and morphology, 275-288. Cambridge, MA: MIT Working Papers in Liguistics.

Haugen, Jason D. \& Daniel Siddiqi. 2016. Towards a restricted realization theory: Multimorphemic monolistemicity, portmanteaux, and post-linearization spanning. In Daniel Siddiqi \& Heidi Harley (eds.), Morphological metatheory, 343-386. Amsterdam: John Benjamins. https://doi.org/10.1075/la.229.12hau.

Iatridou, Sabine, Elena Anagnostopoulou \& Roumyana Izvorski. 2001. Observations about the form and meaning of the Perfect. In Michael Kenstowicz (ed.), Ken Hale: A life in language, 189-238. Cambridge, MA: MIT Press.

Merchant, Jason. 2001. The syntax of silence: Sluicing, islands, and the theory of ellipsis (Oxford Studies in Theoretical Linguistics 1). Oxford: Oxford University Press.

Merchant, Jason. 2018. Ellipsis: A survey of analytical approaches. In Jeroen van Craenenbroeck \& Tanja Temmerman (eds.), The Oxford handbook of ellipsis, 18-45. Oxford University Press. https://doi.org/10.1093/oxfordhb/9780198712398.013.2.

Murphy, Andrew. 2016. Subset relations in ellipsis licensing. Glossa: A Journal of General Linguistics 1(1). 44. https://doi.org/10.5334/gjgl.61.

Noyer, Rolf. 1992. Features, positions and affixes in autonomous morphological structure. Cambridge, MA: Massachusetts Institute of Technology dissertation.

Ostrove, Jason. 2018. Stretching, spanning, and linear adjacency in Vocabulary Insertion. Natural Language \& Linguistic Theory 36(4). 1263-1289. https://doi.org/10.1007/s11049-018-9399-y.

Radkevich, Nina. 2010. On Location: The structure of case and adpositions. Storrs, CT: University of Connecticut dissertation.

Ronai, Eszter \& Laura Stigliano. 2020. NP ellipsis bleeds allomorphy in Hungarian. Snippets 39. 10-12. https://doi.org/10.7358/snip-2020-039-rost.

Sailor, Craig. Forthcoming. The morphophonology of ellipsis: Evidence for Segregated Transfer. In Anikó Lipták \& Güliz Günes (eds.), The derivational timing of ellipsis. Oxford: Oxford University Press.

Svenonius, Peter. 2016. Spans and Words. In Heidi Harley \& Daniel Siddiqi (eds.), Morphological metatheory, 199-220. Amsterdam: John Benjamins.

Travis, Lisa. 1984. Parameters and effects of word order variation. Cambridge, MA: Massachusetts Institute of Technology dissertation.

Trommer, Jochen. 1999. Morphology consuming syntax' resources: Generation and parsing in a Minimalist version of Distributed Morphology. Manuscript.

Weisser, Philipp. 2019. Telling allomorphy from agreement. Glossa: A Journal of General Linguistics 4(1). 86. https://doi.org/10.5334/gjgl.803. 